Journal of English Language Teaching and Applied Linguistics

ISSN: 2707-756X

DOI: $10.32996 /$ jeltal

Journal Homepage: www.al-kindipublisher.com/index.php/jeltal

\title{
Language and the Brain: A Twofold Study of Language Production and Language Comprehension as a Separate or Integrated Set of Processes
}

\author{
Anokye Bernice 8(D) \\ School of Foreign Language and Literature, Nanjing Tech University, Nanjing, China \\ $\square$ Corresponding Author: Anokye Bernice, E-mail: berniceanokye91@gmail.com
}

\section{ARTICLE INFORMATION}

Received: April 14, 2021

Accepted: May 18, 2021

Volume: 3

Issue: 5

DOI: $10.32996 /$ jeltal.2021.3.5.9

\section{KEYWORDS}

Psycholinguistics, production, comprehension, language and the brain

\section{ABSTRACT}

Humans can understand their language due to the processes in the brain. It is very easy for language users to presume that language production and language comprehension are two simple phenomena. For psycholinguistics, these two processes are part of the three core topics in the study of the language and the mind. Psycholinguistics attempt to have a model that explains how language is processed in our brain. It is nearly impossible to do or think about anything without using language, whether this entails following a set of written instructions or an internal talk-through by your inner voice. Language permeates our brains and our lives like no other skill. Beforehand, psycholinguists described our comprehension and production of language in terms of the rules that were hypothesized by linguists (Fodor, Bever, \& Garrett, 1974). Now, that is not the case. These linguistic rules inform rather than taking precedent in studying language and the brain. This paper aims to describe the brain regions/structures, language processes, and the intricate connections between them. The study discusses the brain as the underlying basis of the relationship between language and the brain. Moreover, this study descriptively analyses some of the recent expositive psycholinguistic research on language production and comprehension in order to understand the nature and dynamics of language. The methodology of this paper has to do with the research design, materials and concludes with descriptive analyses of the major finding from the secondary data reviewed in the paper. The linguistic approaches used for this study do not entail any sort of calculation or enumeration. It takes the form of a descriptive qualitative approach or a desktop study where research work mainly capitalizes on preexisting literature in the research domain. The study's main finding reveals that research works on language processing treat production and comprehension as quite distinct from each other. Language production processes differ fundamentally from comprehension processes in many respects. However, other researchers reject such a dichotomy. In its place, they propose that producing and understanding are tightly interwoven, and this interweaving underlies people's ability to predict themselves and each other.

\section{Introduction}

Language may be defined as a system of symbols with commonly recognized meanings which makes easier our thought processes and helps us to communicate with each other. An increasing number of psychologists have devoted themselves to the study of language recently due to the growing interest in the field of communication. Language production and comprehension are among the most automatic tasks humans perform. Yet, they are also the most complex; Language production primarily focuses on the formulation of single, isolated utterances. An utterance is usually made up of one or more words, spoken together under a single intonational contour or personifying a single idea (e.g., Boomer, 1978; Ferreira, 1993), while comprehension requires the simultaneous integration of many different types of information, such as knowledge about

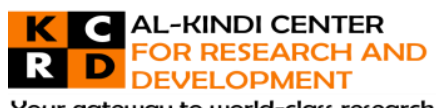

Your gateway to world-class research

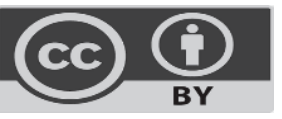

Published by Al-Kindi Center for Research and Development, United Kingdom. Copyright (c) the author(s). This open access article is distributed under a Creative Commons Attribution (CC-BY) 4.0 license 
alphabets or letters and their sounds, spelling, grammar, word meanings, and general world knowledge. To add, general cognitive abilities such as attention monitoring, inferencing, and memory retrieval are used in order to organize this information into a single meaningful representation.

Psycholinguistics as an interdisciplinary field has become the focus of researchers who study the interrelation between the mind and language. Psycholinguistics means the psychology of language, which is studying the psychological and neurological factors that enable humans to acquire, use, comprehend, and produce language ("Altman", 2001, p.1). It embodies how language and speech are acquired, produced, comprehended and lost.

Early psycholinguists described language comprehension and production in terms of the rules hypothesized by linguists (Fodor, Bever, \& Garrett, 1974). The connections between linguistics and psychology were relatively close in the area of syntax, with psycholinguists testing the psychological reality of various proposed linguistic rules. As the field of psycholinguistics developed, researchers became aware that theories of sentence comprehension and production cannot be based simply on any linguistic theories. It was pertinent that psycholinguistic theories consider the properties of the human mind as well as the structure of the language. However, psycholinguistics has since become an area of inquiry on its own, however, informed by but not totally dependent on linguistics. Psycholinguistics is thus the field of language studies that focuses on the psychological processes involved in how language is used, including language production, comprehension and the acquisition of the first and or second language.

For a skilled language user, understanding and producing language seem deceptively simple. For a psycholinguist, language comprehension and production is a complex interaction of various processing components, which include accessing the lexicon, building a syntactic structure, encoding and decoding the sound patterns of a language, and interpreting and expressing intended pragmatic messages. By studying these various components, psycholinguists attempt to figure out what processes, mechanisms, or procedures underlie language use and learning.

According to Levelt, language production is logically divided into three major steps, including deciding what to express (conceptualization), determining how to express it (formulation), and expressing it (articulation; Levelt, 1989). Comprehension can be said to be the sense that a listener feels from the speaker, takes the speaker's interpretation, puts it away in mind, cultivates it, and concludes with the suspense, whether good or bad.

Language and the brain have many complex interrelating elements and to gain a deeper understanding of Psycholinguistics, we must examine this relationship. To study how the brain processes language, there are a number of sub-disciplines with noninvasive techniques for studying the neurological undertaken of the brain. For example, neurolinguistics has become a field in its own right. Psycholinguistics takes into account the cognitive processes that make it possible to generate grammatical and meaningful sentences out of vocabulary and grammatical structure, as well as the processes that make it possible to understand utterances, words, texts, etc. (Miller \& Emas, 1983).

This paper aims to describe the various brain regions/structures, language processes, and the intricate connection between them. The brain will be explored, as it is the core element in the relationship between language and the brain. In order to understand the nature and dynamics of language, we must understand how psycholinguists interpret the brain and how it relates to language processes.

In this paper, a selective review of some recent illustrative psycholinguistic research on language production, comprehension has also been made.

From the perspective of the language producer (speaker, writer), the production of a message takes us from an underlying intention, through stages of planning sentence structures and selecting words, to the articulation of that intention as a sequence of sounds or letters. From the comprehender's (listener's, reader's) perspective, the goal is to perceive or recognize elements such as letters and sounds in the input and work out the connections between these words in sentence structures to arrive at a message-level interpretation.

\section{Literature Review}

This section expounds on the existing literature on psycholinguistics, language production and language comprehension. It also reviews how psycholinguist interprets the interrelation between the mind and language.

\subsection{Psycholinguistics}

According to the Wikipedia-world wide web free encyclopedia, psycholinguistics is the sub-field of cognitive psychology that studies the psychological basis of linguistic competence and performance. It studies the neurological and psychological factors that enable humans to acquire, use, and understand language. Psycholinguistics mainly concern the use of psychological / 
scientific / experimental methods to study language acquisition, production and processing. Psycholinguistics is in short a scientific study of mental processes and elements employed in language use.

One can also explain psycholinguistics as the theoretical and empirical study of the mental faculty. Ever since the linguistic revolution of the mid-1960s, the field of psycholinguistics has developed to encompass a wide range of topics and disciplines. As did the rest of psychology, psycholinguistics started in the early to mid1960s. The revolution termed as the Chomskian revolution (e.g. Chomsky, 1957, 1965, and 1968) promoted language and specifically its structure, as obeying laws and principles in much the same way as say chemical structures do.

The field has been developed and redefined by the reaction to Chomsky. Chomsky's argument about creating syntactic sentences was that language exists because humans possessed an innate ability and were highly critical of skinners book in 1959. This review began what has been dubbed 'the cognitive revolution in psychology. According to Anderson, the review of Chomsky still holds that the human ability to use syntax is qualitatively different from any sort of animal communication; this ability may have resulted from an adaptation of skills evolved for other purposes (Anderson, 1998). It is very easy for language users to presume that language production and language comprehension are two simple phenomena. For psycholinguistics, these two processes are part of the three core topics in the study of language and the mind.

They typically study language comprehension and production as separate sets of processes. The language production system is tasked with translating thoughts and desires into a motor plan for action, moving through word selection, syntactic planning and phonological planning stages along the way. The comprehension system is charged with a different task. It is tasked to take as input an auditory or visual signal, identify the words in that signal and assign the input a structure and a meaning.

However, researchers have the right reasons to view production and comprehension as parts of one language system. One reason for this is that comprehension and production both pose distinct challenges to language users. Case in point, language comprehension involves extracting meaning from a speech signal or printed text, whereas language production involves converting a preverbal message into speech or text using appropriate lexicon, grammar, and phonology or orthography. Another reason is that compared to comprehension, production appears to be much harder to study experimentally. This is because researchers often find it difficult to control input and elicit relevant output when studying language production.

\subsection{Language production}

According to Levelt (1989), language production is logically divided into three major steps:

1) deciding what to express (conceptualization),

2) determining how to express it (formulation), and

3) expressing it (articulation).

Albeit; achieving conversational goals, structuring of narratives, and modulating the ebb and flow of dialogue are inherently important to understanding how people speak (Clark, 1996), psycholinguistics study of language production has primarily focused on the formulation of single, isolated utterances. An utterance consists of one or more words, spoken together under a single intonational contour or expressing a single idea (e.g., Boomer, 1978; Ferreira, 1993).

According to Griffin and Ferreira (2006), there are three sorts of mental processes;

Conceptualizing Starting with some notion or abstract idea of what we want to say (about the world, the current situation)

Formulating Putting together the elements of language to express the idea, drawing on knowledge of our language, including grammar and the lexicon.

\section{Types of a slip of the tongue}

These errors are bound to appear at all levels of formulating (from phoneme, morpheme to word level).

\begin{tabular}{|l|l|}
\hline Type & Example \\
\hline Shift & That's so she'll be ready in case to hits it (decides to hit it) \\
\hline exchange & Fancy getting your model renosed (getting your nose remodeled) \\
\hline
\end{tabular}




\begin{tabular}{|l|l|}
\hline Anticipation & fake my bike (take my bike) \\
\hline Perseveration & He pulled a pantrum (tantrum) \\
\hline Addition & She didn't explain this clarefully enough (carefully enough) \\
\hline Deletion & I'll just get up and mutter intelligible (unintelligibly) \\
\hline Substitution & At low speeds it's too light (heavy) \\
\hline Blend & That child is looking to be spaddled (spanked/paddled) \\
\hline
\end{tabular}

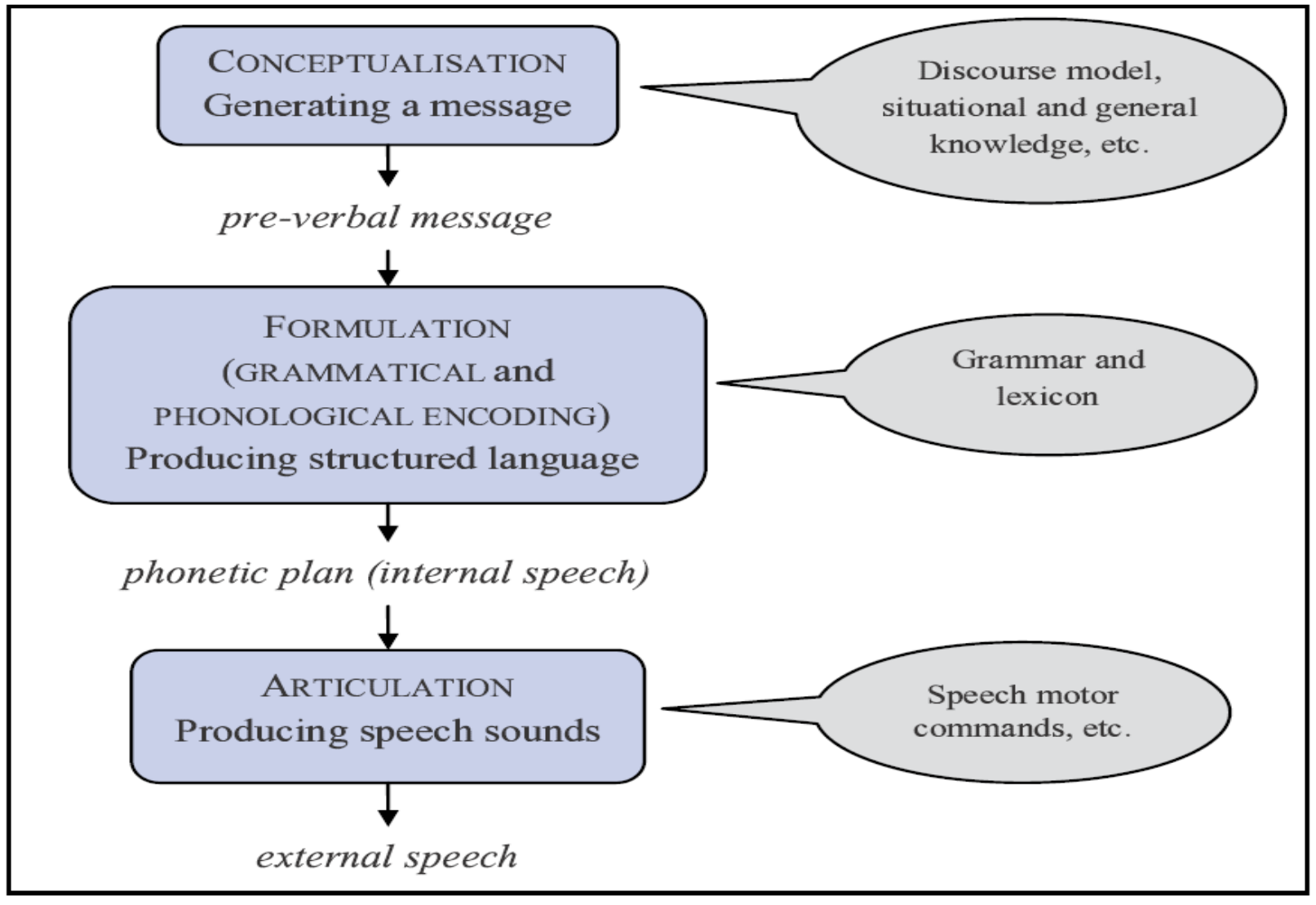

Figure 1: A sketch of the production process

Articulating; speaking this utterance, involving our speech material

The conceptualization stage might pompously perceive itself as the primary and ultimate composer of communication. The formulation stage might take pride in it being a conductor and orchestrator of speech sounds. The articulation stage might regard itself as the instruments of the music of our voices.

\section{Language production}

While Ferreira and Englehart's view on syntax describes processes that allow speakers to produce their words in grammatical utterances, this paper focuses instead on processing the words themselves. Unarguably, theories of multi-word utterance or sentence production fundamentally sum up to an account of how sentences obtain their word orders and structures, how the dependencies between words are accommodated (e.g., subject-verb agreement), and a functionally independent account of how individual content words are generated (e.g., Chang, Dell, Bock, \& Griffin, 2000; Ferreira, 2000; Kempen \& Hoenkamp, 1987). 
The study of language production has primarily focused on the formulation of single, isolated utterances. An utterance consists of one or more words, spoken together under a single intonational contour or expressing a single idea (e.g., Boomer, 1978; Ferreira, 1993).

The first topic is how to generate words. The simplest meaningful utterance consists of a single word. Generating a word begins with specifying its semantic and pragmatic properties. That is, a speaker decides upon an intention or some content to express (e.g., the desired outcome or an observation) and encodes the situational constraints on how the content may be expressed (e.g., polite or informal speech, monolingual or mixing languages; see Levelt, 1989). This process, termed conceptualization or message planning, is traditionally considered prelinguistic and language-neutral (Garrett, 1975; Levelt, 1989). However, speakers may include different information in their messages when preparing to speak different languages (see Slobin, 1996, on thinking for speaking).

The formulation comes after generating words. It is divided into word selection and sound processing stages (Fromkin, 1971; Garrett, 1975). Deciding which word to use mostly include selecting a word in one's vocabulary based on it corresponding to semantic and pragmatic specifications. The significant word representation has often termed a lemma (Kempen \& Huijbers, 1983), lexical entry, lexical representation, or clearly a word, and it targets the presence of a word in a speaker's vocabulary that is capable of expressing particular semantic and pragmatic content within a particular syntactic context. In contradiction, sound processing involves constructing the phonological form of a selected word by retrieving its individual sounds and organizing them into stressed and unstressed syllables (phonological encoding) and then specifying the motor programs to realize those syllables (phonetic encoding). The last process is articulation, which is the discharge of motor programs to pronounce the sounds of a word.

As a matter of interest, recent models of word production agree on the basic facts about how the system works to a great extent, with very least minor variations in explanatory mechanisms. When models differ, the tendency is concerned with the different stages of production, such as word selection or phonological encoding, and different aspects of these stages, such as speed of processing or how processing may go awry to yield speech errors. This means that of the properties of production described below, most are accounted for (at least to some level of detail) by most models of production.

\subsection{Language comprehension}

During the last half of the 20th century, psycholinguists focused on speech perception stood fairly distinct from the research on audition and other modalities of high-level perception such as vision. Current research, however, is beginning to bridge this traditional divide. Fundamental principles that govern all perception, some known for more than a century, are most likely shaping our comprehension of perception of speech in addition to other familiar sounds.

Researchers of speech perception traditionally attempted to investigate how listeners perceive the spoken acoustic signal as a sequence of consonants and vowels, collectively referred to as phonetic segments or units. When speech sounds are described in this way, brackets are used to surround phonetic symbols such as [j] (the ' $y$ ' sound in 'yes') and [o] (as in 'oh'). Contrary to this, phonemes are more abstract linguistic units that roughly correspond to letters in written language and are transcribed surrounded by slashes (/j/ and /o/.) Morphemes are the smallest meaningful units of language, roughly corresponding to words (e.g., 'cat', 'taste', as well as 'dis'- and - 'ful') with phonemes being the smallest units that can change the meaning of a morpheme (e.g., 'yo' versus 'go') (Trubetskoy, 1969). Within this design, the experimental study of speech perception classically has corresponded more or less to the lowest division of labor generally agreed upon by linguists and psycholinguists.

To the extent that speech perception researchers' task is to deliver minimal units to those who study language, an important caveat must be applied to this inherited division of labour. Their experimental evidence demonstrating that either phonetic segments or phonemes are real outside of linguistic theory is unclear (e.g., Lotto, 2000), and the appeal of phonetic segments and phonemes may arise principally from experience with alphabetic writing systems (e.g., Morais, Bertelson, Cary, \& Alegria, 1986; Morais, Cary, Alegria, \& Bertelson, 1979; Port, in press). A person should not be sanguine about whether speech perception is about recognizing consonants and vowels per se. Listeners presumably do not extract phonemes preliminary to recognizing words. There is no evidence of an area in the brain where phonemes reside independently of the words they are made up of.

Nonetheless, conceptualizing speech perception as a process by which phonemes are retrieved from acoustic signals is tradition. Here, research in speech perception often focuses on segmentation problems and lack of invariance. The segmentation problem refers to the fact that, if phonetic units exist, they are not like typed letters on a page. Rather, they overlay broadly in time, much like cursive handwriting. The problem of lack of invariance (or variability) is related to the segmentation problem. Since speech sounds are produced such that its neighbors dramatically colour articulations for one consonant or vowel overlaps with the production of preceding ones, and contrariwise, every consonant and vowel produced in fluent connected speech. Some of the most obstreperous problems in the study of speech perception are as a result of adopting discrete phonetic units as a level of 
analysis, a level that is not discrete and may not be real. In connected speech, the acoustic realization of the beginning and end of one word also overlaps with sounds of preceding and following words, so the problems of invariance and segmentation are not restricted to phonetic units.

\subsection{Comprehension of sounds}

Phoneme restoration effect refers to a device by psycholinguist to investigate comprehension; e.g. subjects hear 'ail' in a paragraph describing how water is gathered and believe that they heard the word 'pail'. People seek contextual consistency and plausibility, even if it comes to adding a sound or inventing a word that was not actually spoken.

Firstly, people do not necessarily hear each of the words spoken to them. Comprehension is not the passive recording of whatever is hear or see.

Secondly, comprehension is strongly influenced by even the slightest changes in discourse that the listener is attending to.

Finally, comprehension is not a simple item-by-item analysis of words in a linear sequence.

Voice Onset Timing (VOT) refers to the brief burst of air that precedes all stop consonants' articulation, which provides phonetic information listeners use to distinguish between sound like $/ \mathrm{k} /$ and $/ \mathrm{g} /$.

The acquisition of this phonetic ability cannot be completely explained only by exposure to or instruction in the language up to now. The ability to focus on VOT differences is innate. We categorize these minute phonetic differences in a non-continual binary fashion.

Categorical perception refers to listening to a stream of speech and automatically dividing this continuous flow of sound into the phonemes of the listener's native language, which is seemingly unique to human beings and appears to qualify as one aspect of UG, the genetic propensity for comprehending and producing language.

Categorical perception is the most well-known pattern of perceptual performance with speech sounds. Three common features define categorical perception: sharp labeling (identification) function, discontinuous discrimination performance (near-perfect across identification boundary and near-chance to either side), and the ability to predict discrimination performance purely based on labelling data (Wood, 1976). All of these three defining markers or features of categorical perception arise naturally from the principle of discovering (and continuously absorbing) predictability in the interest of maximizing sensitivity to change.

\subsection{Language and the human brain}

Psycholinguistics is an interdisciplinary field and recently, researchers who study the interrelation between the mind and language are interested. It studies how language and speech are acquired, produced, comprehended and lost. Whereas language acquisition and language dissolution happen diachronically or overtime, language production and language comprehension happen at a certain point in time or simultaneously. In other words, psycholinguistics means the psychology of language studies and the psychological and neurological factors that enable humans to acquire, use, comprehend and produce language ("Altman", 2001, p.1). This is mainly due to a lack of cohesive data on how the human brain functions. Modern research makes use of natural science concerned with the study of life and living organism (biology), the scientific study of how information concerning faculties such as perception (neuroscience), language, reasoning, and emotion is represented and transformed in a human or another animal nervous system or machinery, linguistics and a branch of applied mathematics and electrical engineering involving the quantification of information (information theory).

Language processing is an inherent characteristic of humans. The knowledge about its neurobiological basis has been improved considerably over the past decades. The study of brains has led researchers to identify and understand that different regions in the brain's left and right hemisphere support particular language functions. Networks and connections involving the temporal cortex and the inferior frontal cortex with clear left lateralization were shown to support syntactic processes, whereas less lateralized temporo-frontal networks sub-serve semantic processes. These networks have been verified both by functional as well as by structural connectivity data. Electrophysiological measures show that within these networks, the syntactic processes of local structure building precede grammatical and semantic relations in a sentence. Suprasegmental prosodic information obviously available in the acoustic language input is processed predominantly in a temporo-frontal network in the right hemisphere associated with a clear electrophysiological marker. Language as a system is controlled by the brain that is different from but closely linked to general cognition. There are two hemispheres in the human brain. The left hemisphere is the "logical brain" and is involved in language and analysis and the right hemisphere is said to be the "creative brain," involved in daydreaming and imagination. The left hemisphere controls the right side of the body, whereas the right hemisphere controls the left side. The earliest research on speech and language centers of the brain dates back to the early nineteenth century. Doctors asserted that patients who had brain-injuries with damages to the left hemisphere tend to lose power of speech and language abilities, while those with injuries to the right hemisphere did not lose this ability. 
Language and the brain are complexly related and in order to gain a deeper understanding of Psycholinguistics, we must examine this relationship. To study how the brain processes language, there are a number of sub-disciplines with non-invasive techniques for studying the neurological workings of the brain. For instance, neurolinguistics has become a field in its own right. Psycholinguistics focus on the cognitive processes that help generate grammatical and meaningful sentences out of vocabulary and grammatical structure and the processes that make it possible to understand utterances, words, texts, etc. (Miller \& Emas, 1983).

This is vital to modern linguistics; human language is a natural object (our species)-specific ability to acquire a language, our implicit knowledge of the immense complexity of language, and our competence to use language in open, fitting, and inexhaustible ways are attributed to a property of the natural world, our brain. The brain is composed of neurons, nerve cells that are the basic information processing units of the nervous system.

Language is a function of the human brain structure, and several brain regions have been identified with linguistic capabilities. Due mainly to a lack of cohesive data on how the human brain functions, modern research uses biology, neuroscience, language, reasoning, and emotion to be represented and transformed in a human or another animal nervous system or machinery, linguistics, and information theory. Psycholinguists particularly is concerned with the theories behind language acquisition and how the mind aids in a person's ability to gain language. During the 1950's, a famous linguist, Noam Chomsky developed a theory known as Nativism. This theory substantiates the fact that children will never gain the resources needed for processing language just by the things they heard, but more by how the language acquisition device works (Ambridge $\&$ Lieven, 2011). This theory advocated that all people are born with a universal grammar that grants them the ability to acquire language. Psycholinguistics attempts to carve out a model that represents how language is processed in the brain. It is nearly hopeless to do or think about anything without using language. Whether this subsumes an internal talk-through by a voice inside one's head or following a set of written instructions, language permeates our brains and lives like non-other skills. For what looks like more than a century, it's been accepted that our capacity to use language is usually located in the left hemisphere of the brain, specifically in two areas: Broca's area (associated with speech production and articulation) and Wernicke's area (associated with comprehension). Hence any harm to either of these, caused by a person stroking out or blunt force trauma or another injury, can lead to language and speech problems or aphasia; a language deficit caused by damage to the brain, often be a stroke or an accident.

\section{Methodology}

This chapter is concerned with the research design, materials and concludes with descriptive analyses of the major finding from the secondary data reviewed in the paper. It is worth noting that the linguistic approaches adopted for this study do not involve any sort of calculation or enumeration. It takes the form of a descriptive qualitative approach or a desktop study where research work mainly capitalizes on preexisting literature in the research domain.

\subsection{Research design and materials}

The research design adopted for this study is a desktop research design. All data collected were already existing in print or published on the internet. The material used in this study is fundamentally secondary data.

\subsection{Major Findings}

From various research works on language production and comprehension, the research arrived at this major finding:

1. It is confirmed that language resides in the left hemisphere of the brain. While language acquisition and language dissolution happen over time or diachronically, language production and language comprehension happen at a certain point in time.

2. It is easy for language learners and researchers to think of production and comprehension as parts of one language system. This is because comprehension and production both pose distinct challenges to language users. For example, language comprehension involves extracting meaning from a speech signal or printed text, whereas language production involves converting a preverbal message into speech or text using appropriate lexicon, grammar, and phonology or orthography. Another reason is that compared to comprehension, production appears to be much harder to study experimentally. This is because researchers often find it difficult to control input and elicit relevant output when studying language production.

3. Comprehension goes beyond the passive recording of whatever is heard or seen. It can be said to be the sense that a listener feels from the speaker, takes the speaker's interpretation, puts it away in mind, cultivates it, and concludes with the suspense, whether good or not. 
4. The core interest of cognitive psychology is understanding the nature of the mental representation of information and the processes that perform a function on those representations.

5. Language is a function of the human brain structure, and several brain regions have been identified with linguistic capabilities.

6. Evidence for speech production behavior has until recently relied heavily on speech errors (laboratory-induced or natural), and speech errors are not made at random

\section{Results and Discussion}

Psycholinguistics is the discipline that studies the linguistic performance of speech production and comprehension. Early psycholinguists described our comprehension and production of language in terms of the rules that were postulated by linguists (Fodor, Bever, \& Garrett, 1974). The link between psychology and linguistics was close in syntax in particular, with psycholinguists testing the psychological reality of various proposed linguistic rules. As psycholinguistics developed into a comprehensive research field, it became obvious that theories of sentence comprehension and production cannot be simply based on linguistic theories; psycholinguistic theories ought to consider the properties of the human brain well as the structure of the language. As explained earlier in this paper, psycholinguistics has since become an independent area of inquiry, thus informed by and not totally dependent on linguistics.

The relationship between language and the human brain is symmetrical. Language is a function of the human brain structure, and several brain regions have been identified with linguistic capabilities. As a result of the lack of cohesive data on how the human brain functions, modern research uses biology, neuroscience, language, reasoning, and emotion to be represented and transformed in a human or other animal nervous system or machinery, linguistics, and information theory. Psycholinguists are particularly concerned with the theories behind language acquisition and how the mind aids in a person's ability to gain language.

Furthermore, the fundamental concerns of cognitive psychology are understanding the nature of the mental representation of information and the processes that operate on those representations have received their most extensive examination in the past decade in the field of psycholinguistics. The reasons for this are evident; language is, to a degree, an accessible field of study, and the systematic classification and observation brought by linguistics and philosophy to the language provided a primary initial bedrock and advancement for the investigation of the psychological functions rudimental to human language production and comprehension. And while there has been much progress in several other fields in the area of cognition, the now advanced field of psycholinguistics still represents one of the most promising and profitable areas for examining cognitive function. It appears seemingly that enormous breakthroughs in understanding the extent of (and constraints on) our ability to perceive, process, store, recall and comprehend information will come from gaining a detailed empirical understanding of the nature of language processing.

Pre-modern views of language studies presume separate processing "streams" for language production and comprehension. They adopt the "cognitive sandwich," a conflicting perspective, both with the demands of communication and extensive data indicating that production and comprehension are tightly interrelated.

Production processes must, of course, be used when individuals produce language, and comprehension processes must be used when they comprehend language. However, production processes must also be used during, for example, silent naming, when no utterance is produced. Silent naming, therefore, involves some production processes (e.g., those associated with aspects of formulation such as name retrieval) but not others (e.g., those associated with articulation; see Levelt 1989). Likewise, comprehension processes must occur when a participant retrieves the phonology of a masked prime word but not its semantics (e.g., Van den Bussche et al. 2009). And so, it is also possible that production processes are used during comprehension and comprehension processes used during production.

An integrated or separate theory of language production and comprehension

Current accounts of language processing treat production and comprehension as quite distinct from each other. The split is clearly reflected in the structure of recent handbooks and textbooks concerned with the psychology of language (e.g., Gaskell 2007; Harley 2008). This structure does not merely reflect organizational convenience but instead treats comprehension and production as two different questions to investigate. For example, researchers assume that the processes involved in comprehending a spoken or written sentence, such as resolving ambiguity, may be quite distinct from the processes involved in producing a description of a scene. In neurolinguistics, the "classic" Lichtheim-Broca-Wernicke model assumes distinct anatomical pathways associated with production and comprehension, primarily on the basis of deficit-lesion correlations in aphasia (see Ben Shalom \& Poeppel 2008). However, other researchers reject such a dichotomy. In its place, propose that 
producing and understanding are tightly interwoven, and this interweaving underlies people's ability to predict themselves and each other.

\section{Conclusion}

In conclusion, it is very easy for language users to presume that language production and language comprehension are two simple phenomena. For psycholinguistics, these two processes are part of the three core topics in the study of language and the mind. Early century accounts of language presuppose separate processing "streams" to produce and comprehend language in the brain. They espouse the "cognitive sandwich," a perspective that is incompatible both with the demands of communication and with extensive data indicating that production and comprehension are tightly interwoven.

Language processing is a trait of the human species and current accounts of language processing treat production and comprehension as quite distinct from each other. The split is clearly reflected in the structure of recent handbooks and textbooks concerned with the psychology of language (e.g., Gaskell 2007; Harley 2008). This structure does not merely reflect organizational convenience but instead treats comprehension and production as two different questions to investigate.

\section{References}

[1] Anderson, J. A., \& Rosenfeld, E. (Ed.) (1998). Talking nets: An oral history of neural networks. Cambridge, MA: MIT Press

[2] Altmann T. M. Gerry (2001), Psycholinguistics in review British Journal of Psychology

[3] 129-170 printed in Great Britain the British Psychological Society.

[4] Boomer, D. S. (1978). The phonemic clause: Speech unit in human communication. In: A. W. Seigman \& S. Feldstein (Eds.), Nonverbal behavior and communication (pp. 245-262). Hillsdale, NJ: Erlbaum.

[5] Chomsky, N. (1957). Syntactic structures. The Hague: Mouton.

[6] Chomsky, N. (1959). Review of Skinner's Verbal behavior. Language, 35, $26-58$.

[7] Chomsky, N. (1965). Aspects of the theory of syntax. Cambridge, MA: MIT Press. Chomsky, N. (1968). Language and mind. New York: Harcourt Brace.

[8] Chang, F., Dell, G. S., Bock, K., \& Griffin, Z. M. (2000). Structural priming as implicit learning: A comparison of models of sentence production. Journal of Psycholinguistic Research, 29, 217-229.

[9] Ferreira, F. (1993). The creation of prosody during sentence production. Psychological Review, 100, 233-253.

[10] Fromkin, V. A. (1971). The non-anomalous nature of anomalous utterances. Language, 47, $27-52$.

[11] GARNHAM, A. (1985). Psycholinguistics: Central topics. London: Methuen

[12] Harley, T. (2005): The Psychology of Language. From Data to Theory, New York,

[13] Psychology press Translation"vol. 52, p. 13-21.

[14] Kempen, G., \& Hoenkamp, E. (1987). An incremental procedural grammar for sentence

[15] Levelt, W. J. M. (1989). Speaking: From intention to articulation. Cambridge, MA: MIT Press formulation. Cognitive Science, 11, $201-258$.

[16] Slobin, D. I. (1996). From "thought and language" to "thinking for speaking". In: J. Gumperz \& S. C. Levinson (Eds), Rethinking linguistic relativity: Studies in the social and cultural foundations of language (pp. 70-96). New York: Cambridge University Press. 